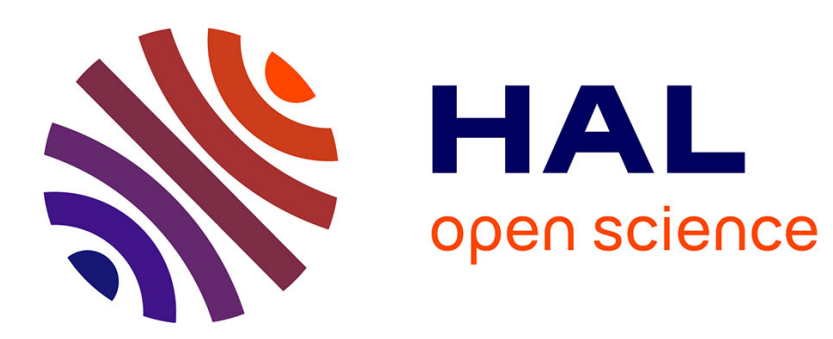

\title{
Génération de bruit par appariement de tourbillons dans les zones cisaillées
}

Christophe Bogey, Christophe Bailly

\section{To cite this version:}

Christophe Bogey, Christophe Bailly. Génération de bruit par appariement de tourbillons dans les zones cisaillées. Mécanique et Industries, 2000, 1 (5), pp.487-497. hal-02353101

\section{HAL Id: hal-02353101 \\ https://hal.science/hal-02353101}

Submitted on 7 Nov 2019

HAL is a multi-disciplinary open access archive for the deposit and dissemination of scientific research documents, whether they are published or not. The documents may come from teaching and research institutions in France or abroad, or from public or private research centers.
L'archive ouverte pluridisciplinaire HAL, est destinée au dépôt et à la diffusion de documents scientifiques de niveau recherche, publiés ou non, émanant des établissements d'enseignement et de recherche français ou étrangers, des laboratoires publics ou privés. 
Mec. Ind. (2000) 1, 487-497

(C) 2000 Éditions scientifiques et médicales Elsevier SAS. Tous droits réservés

S1296-2139(00)01053-8/FLA

\title{
Génération de bruit par appariement de tourbillons dans les zones cisaillées
}

\author{
Christophe Bogey *, Christophe Bailly \\ LMFA, UMR CNRS 5509, École Centrale de Lyon, BP 163, 69131 Ecully cedex, France
}

(Reçu le 30 mars 2000 ; accepté le 1 septembre 2000)

\begin{abstract}
Résumé - On étudie la génération de bruit dans une couche de mélange subsonique bidimensionnelle. Afin de calculer le rayonnement acoustique directement par la résolution des équation de Navier-Stokes filtrées, on utilise un code de simulation des grandes échelles (ALESIA pour Appropriate Large Eddy SImulation for Aeroacoustics) construit avec des techniques numériques spécifiques à l'aéroacoustique, en particulier au niveau des conditions aux limites. La couche de mélange est excitée à sa fréquence fondamentale et à son premier sous-harmonique pour fixer le lieu des appariements de tourbillons. Les fusions successives produisent un rayonnement acoustique à la fréquence de ces appariements, et le caractère de quadrupôle tournant de la source de bruit associée à deux tourbillons co-rotatifs est mise en évidence. La dynamique des appariements est également analysée pour expliquer le rayonnement acoustique global obtenu. (c) 2000 Éditions scientifiques et médicales Elsevier SAS
\end{abstract}

simulation des grandes échelles (SGE) / aéroacoustique numérique / couche de mélange / appariement de tourbillons / quadrupôle tournant

Abstract - Noise generation by vortex pairings in shear flows. Noise generation in a bidimensional subsonic mixing layer is investigated. In order to obtain the acoustic radiation directly from the filtered Navier-Stokes equations, a large eddy simulation code (ALESIA, Appropriate Large Eddy SImulation for Aeroacoustics) is built up using specific numerical techniques of computational aeroacoustics, and especially for boundary conditions. The mixing layer is excited at its fundamental frequency and its first subharmonic frequency to fix the location of vortex pairings. The successive pairings generate an acoustic radiation at the frequency of pairings. The sound generation mechanism corresponds to the rotating quadrupole associated to two co-rotative vortices. The dynamics of vortex pairings is also analysed to explain the whole acoustic field. (C) 2000 Éditions scientifiques et médicales Elsevier SAS

large eddy simulation (LES) / computational aeroacoustics (CAA) / mixing layer / vortex pairing / rotating quadrupole

\section{Nomenclature}

$\Theta$

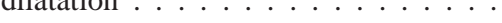

e énergie spécifique interne totale . . . .

$k_{\mathrm{sgs}} \quad$ énergie résiduelle de sous-maille $\ldots . . \mathrm{kg} \cdot \mathrm{m}^{-1} \cdot \mathrm{s}-2$

$\delta_{\omega}(0)$ épaisseur de vorticité de la couche de mélange . . . . . . . . . .

$f_{\mathrm{p}} \quad$ fréquence d'appariements des tourbillons

$f_{0} \quad$ fréquence fondamentale de la couche de mélange . . . . . . . . . . . . .

$\lambda \quad$ longueur d'onde acoustique ......

$\rho$

$$
\text { ........ }
$$$$
\text { masse volumique }
$$

* Correspondance et tirés à part : christophe.bogey@ec-lyon.fr
$R e_{\omega} \quad$ nombre de Reynolds de la couche de mélange

$T_{\mathrm{p}} \quad$ période d'appariements des tourbillons

p pression ............. $\mathrm{Pa}$

$\tau_{i j} \quad$ tenseur des contraintes visqueuses

$S_{i j}$ tenseur des déformations ....... $\mathrm{s}^{-1}$

$T_{i j} \quad$ tenseur de sous-maille $\ldots \ldots \ldots \mathrm{kg} \cdot \mathrm{m}^{-1} \cdot \mathrm{s}^{-2}$

u vecteur vitesse .......... $\mathrm{m} \cdot \mathrm{s}^{-1}$

$\mu \quad$ viscosité moléculaire ...... $\mathrm{m}^{2} \cdot \mathrm{s}^{-1}$

$\mu_{\mathrm{t}} \quad$ viscosité turbulente ....... $\mathrm{m}^{2} \cdot \mathrm{s}^{-1}$

$V_{\mathrm{g}} \quad$ vitesse de groupe des ondes acoustiques $\mathrm{m} \cdot \mathrm{s}^{-1}$

$U_{1}$ vitesse du fluide lent ....... $\mathrm{m} \cdot \mathrm{s}^{-1}$

$U_{2}$ vitesse du fluide rapide ...... $\mathrm{m} \cdot \mathrm{s}^{-1}$

c vitesse du son ......... $\mathrm{m} \cdot \mathrm{s}^{-1}$ 


\section{INTRODUCTION}

Les écoulements turbulents constituent une source de bruit importante. Ils sont responsables dans le domaine des transports de nuisances sonores, qui doivent désormais respecter des normes de plus en plus sévères. Dans le secteur nucléaire, le rayonnement acoustique produit dans les conduites par des écoulements rapides est susceptible d'endommager gravement les structures par effets résonnants induits. La réduction du bruit d'origine aérodynamique est donc aujourd'hui un enjeu majeur. Il convient par conséquent de développer des outils capables de calculer avec précision les niveaux sonores. C'est l'objet de l'aéroacoustique numérique dont le développement depuis une dizaine d'années est spectaculaire.

Depuis l'analogie de Lighthill [1] proposée en 1952, ce sont essentiellement des méthodes hybrides de calcul de bruit qui ont été utilisées. Elles sont construites sur un calcul en plusieurs étapes. Après avoir déterminé un champ turbulent, on utilise un modèle de termes sources acoustiques combiné avec un opérateur de propagation, pour obtenir le rayonnement acoustique. Cette approche de calcul du bruit a permis de retrouver les lois de puissance associées au rayonnement acoustique des jets par exemple, mais présentent plusieurs inconvénients. Tout d'abord, le champ turbulent est souvent obtenu par une modélisation de la turbulence [2], et ne décrit que partiellement le vrai champ de vitesse responsable du rayonnement acoustique. Les modèles des termes sources sont également toujours discutés aujourd'hui. Les effets de réfraction des ondes acoustiques par l'écoulement sont souvent négligés par ces méthodes. D’une façon plus générale, en séparant le traitement des phénomènes aérodynamiques et acoustiques, des informations sont perdues au niveau des interactions entre les champs aérodynamiques et acoustiques.

Une nouvelle méthode de calcul du bruit, basée sur la résolution des équations de Navier-Stokes compressibles instationnaires, est apparue avec le développement de techniques numériques spécifiques à l'aéroacoustique [3]. Elle consiste à calculer le champ acoustique directement à partir des équations de Navier-Stokes, en déterminant simultanément les fluctuations de nature aérodynamique et les fluctuations de nature acoustique. L'intérêt de ce calcul acoustique direct est de fournir une solution exacte, car aucune hypothèse n'est utilisée au contraire des méthodes hybrides. Il est donc théoriquement possible de déterminer le rayonnement acoustique des écoulements turbulents avec une grande précision. Les premières applications du calcul acoustique direct à des jets supersoniques ou subsoniques à faible nombre

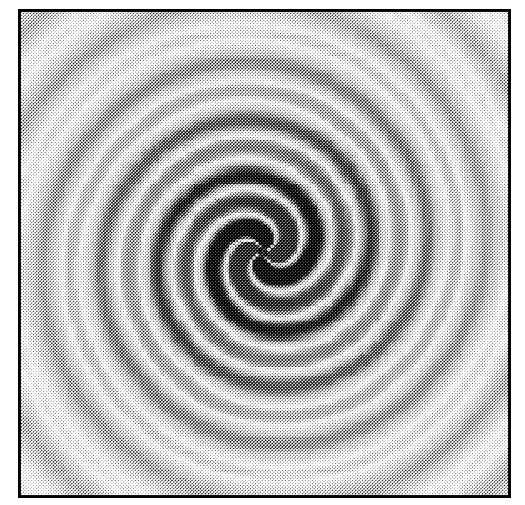

Figure 1. Représentation du champ de dilatation généré par deux tourbillons co-rotatifs.

Figure 1. Snapshot of the dilatation field generated by two corotative vortices.

de Reynolds [4, 5] par simulation numérique directe (SND) ont par exemple fourni des niveaux sonores tout à fait comparables aux données expérimentales correspondantes. Le calcul acoustique direct doit également nous permettre d'étudier de manière assez fine les mécanismes de génération de bruit présents dans les écoulements turbulents, puisque l'on dispose à la fois des champs aérodynamiques et acoustiques.

Les mécanismes de génération de bruit dans les écoulements subsoniques sont encore mal identifiés. On sait depuis les travaux de Powell [6] que l'accélération de la vorticité produit un rayonnement acoustique. On rencontre un tel phénomène notamment lors des interactions des structures tourbillonnaires entre elles ou avec des parois. Le cas de deux tourbillons co-rotatifs est une illustration simple et maintenant assez bien connue de ce mécanisme. Deux tourbillons co-rotatifs placés dans un milieu au repos tournent l'un autour de l'autre pendant une certain temps avant de fusionner. La rotation des tourbillons constitue une source de bruit de type quadrupolaire tournant, dont le rayonnement acoustique associé [7] présente une structure en double hélice comme on le montre sur la figure 1.

Dans cette article, on se propose de simuler une couche de mélange 2-D entre deux écoulements parallèles de vitesse différente, afin de mettre en évidence le rôle des appariements de tourbillons dans le rayonnement acoustique d'une zone de cisaillement. Les expériences de couche de mélange ont montré l'existence de rouleaux tourbillonnaires quasi-bidimensionnels [8]. On peut donc s'attendre à obtenir par notre calcul bidimensionnel le rayonnement acoustique caractéristique d'un tel écoulement. Dans une précédente étude concernant la SND d'une couche de mélange 2-D, Colonius et al. [9] ont 
trouvé un champ sonore rayonné à la fréquence des appariements de tourbillons, en filtrant précisément à cette fréquence. Dans notre étude, l'objectif est de retrouver ce type de rayonnement par simulation des grandes échelles (SGE) sans filtrer le champ acoustique issu de la SGE. Il s'agit également d'analyser le mécanisme de génération de bruit par appariements de tourbillons dans les zones cisaillées de vitesse, en s'appuyant sur les études de ce mécanisme réalisées dans un milieu au repos. Le code ALESIA qui a été développé en 2-D [10], puis en 3-D [11], résout les équations de Navier-Stokes en utilisant un modèle de sous-maille pour simuler les effets des petites structures turbulentes inférieures à la taille de la maille. Afin d'effectuer le calcul direct des fluctuations acoustiques, il inclut des techniques spécifiques à l'aéroacoustique numérique. Le schéma numérique appliqué pour les points intérieurs est exposé en détail dans la deuxième partie. Le traitement des conditions aux limites est crucial en aéroacoustique, et est présenté dans la troisième partie. La quatrième partie est consacrée à la simulation de la couche de mélange et au calcul du rayonnement acoustique produit par les appariements successifs de tourbillons. Le mécanisme de génération de bruit par appariement dans la couche de mélange est alors analysé en détail dans la cinquième partie. Les perspectives de ce travail préliminaire sur la couche de mélange seront données dans la sixieme partie.

\section{ALGORITHME NUMÉRIQUE}

Les équations de Navier-Stokes s'expriment, en coordonnées Cartésiennes, sous la forme conservative suivante, pour une géométrie bidimensionnelle :

$$
\frac{\partial \mathbf{U}}{\partial t}+\frac{\partial \mathbf{F e}}{\partial x_{1}}+\frac{\partial \mathbf{G e}}{\partial x_{2}}-\frac{\partial \mathbf{F v}}{\partial x_{1}}-\frac{\partial \mathbf{G v}}{\partial x_{2}}=0
$$

Le vecteur inconnu $\mathbf{U}$ contient les variables conservatives :

$$
\mathbf{U}=\left(\begin{array}{c}
\rho \\
\rho u_{1} \\
\rho u_{2} \\
\rho e
\end{array}\right)
$$

où $\rho$ représente la masse volumique, $u_{1}$ et $u_{2}$ les deux composantes de la vitesse, et $e$ l'énergie spécifique interne totale. Les flux sont décomposés en flux Eulériens, $\mathbf{F e}$ et $\mathbf{G e}$, et en flux visqueux, $\mathbf{F v}$ et $\mathbf{G v}$. Le système (1) est complété par la définition de l'énergie totale pour un gaz parfait :

$$
\rho e=\frac{p}{\gamma-1}+\frac{1}{2} \rho\left(u_{1}^{2}+u_{2}^{2}\right)
$$

où $\gamma$ est le rapport des chaleurs spécifiques et $p$ la pression. Ainsi, les flux Eulériens sont donnés par les expressions suivantes :

$$
\mathbf{F e}=\left(\begin{array}{c}
\rho u_{1} \\
p+\rho u_{1}^{2} \\
\rho u_{1} u_{2} \\
(\rho e+p) u_{1}
\end{array}\right), \quad \mathbf{G e}=\left(\begin{array}{c}
\rho u_{2} \\
\rho u_{1} u_{2} \\
p+\rho u_{2}^{2} \\
(\rho e+p) u_{2}
\end{array}\right)
$$

et les flux visqueux par :

$$
\mathbf{F v}=\left(\begin{array}{c}
0 \\
\tau_{11} \\
\tau_{12} \\
u_{1} \tau_{11}+u_{2} \tau_{12}
\end{array}\right), \quad \mathbf{G v}=\left(\begin{array}{c}
0 \\
\tau_{21} \\
\tau_{22} \\
u_{1} \tau_{21}+u_{2} \tau_{22}
\end{array}\right)
$$

Le tenseur des contraintes visqueuses $\tau_{i j}$ est défini à partir du déviateur $S_{i j}$ du tenseur des déformations :

$$
\tau_{i j}=2 \mu S_{i j} \quad \text { avec } S_{i j}=\frac{1}{2}\left(\frac{\partial u_{i}}{\partial x_{j}}+\frac{\partial u_{j}}{\partial x_{i}}-\frac{2}{3} \delta_{i j} \frac{\partial u_{k}}{\partial x_{k}}\right)
$$

où $\mu$ est la viscosité dynamique moléculaire.

La résolution directe des équations de Navier-Stokes, appelée simulation numérique directe (SND), impose le calcul de toutes les structures turbulentes, jusqu'à l'échelle de Kolmogorov caractérisant les plus petits tourbillons dissipant l'énergie mécanique. Pratiquement, la limitation de la taille des maillages par les moyens informatiques rend cette méthode applicable uniquement pour des écoulements à bas nombre de Reynolds.

La simulation des grandes échelles (SGE) est une des alternatives à cette méthode pour simuler des écoulements présentant des nombres de Reynolds plus élevés. Elle consiste à calculer uniquement les grosses structures turbulentes, et à modéliser les effets des petites structures non résolues par le maillage. On est alors amené à résoudre les équations de Navier-Stokes filtrées, où l'on note ${ }^{\sim}$ la moyenne de Favre et la moyenne d'ensemble. Un filtrage explicite est appliqué dans l'espace physique à chaque itération, voir l'expression (2). Les coefficients $d_{l}$ du filtre sont choisis pour éliminer les fréquences au delà de la fréquence de coupure du schéma spatial [3]. En 1963, Smagorinsky [12] développa un des premiers modèles de sous-maille, qui reste aujourd'hui encore très utilisé pour les écoulements libres. Le tenseur de sous-maille est modélisé en introduisant une viscosité 
turbulente $\mu_{\mathrm{t}}$ telle que :

$$
T_{i j}=-\bar{\rho} \widetilde{u_{i} u_{j}}+\bar{\rho} \tilde{u}_{i} \tilde{u}_{j}=2 \mu_{\mathrm{t}} \widetilde{S}_{i j}-\frac{2}{3} \bar{\rho} k_{\mathrm{sgs}} \delta_{i j}
$$

où $k_{\mathrm{sgs}}$ est l'énergie cinétique turbulente de sous-maille, encore appelée énergie résiduelle, définie par $\bar{\rho} k_{\text {sgs }}=$ $-T_{\mathrm{kk}} / 2$. La viscosité turbulente $\mu_{\mathrm{t}}$ est construite par analyse dimensionnelle, en formant le produit d'une vitesse caractéristique par une longueur caractéristique. On écrit alors :

$$
\mu_{\mathrm{t}}=\bar{\rho}\left(C_{\mathrm{s}} \Delta_{\mathrm{c}}\right)^{2} \sqrt{2 \widetilde{S}_{i j} \widetilde{S}_{i j}}
$$

où $C_{\mathrm{s}}$ est la constante de Smagorinsky. En imposant à la fréquence de coupure $\pi / \Delta_{\mathrm{c}}$ d'être dans la zone inertielle $\mathrm{du}$ spectre de la turbulence, on détermine alors $\mu_{\mathrm{t}}$ pour avoir une dissipation équivalente à celle que l'on aurait si les petites échelles de la turbulence étaient résolues par le calcul. On trouve alors que $C_{\mathrm{s}} \approx 0,18$. Enfin, pour un maillage anisotrope, on estime usuellement la longueur caractéristique de sous-maille par la relation $\Delta_{\mathrm{c}}=(\Delta x \Delta y)^{1 / 2}$.

L'énergie cinétique de sous-maille $k_{\text {sgs }}$ n'est pas directement calculable à partir du champ résolu, et on supposera dans la suite, en suivant Erlebacher et al. [13] que ce terme est négligeable devant la pression $\bar{p}$. Pour un gaz parfait, on a $T_{\mathrm{kk}}=\gamma M_{\mathrm{sgs}}^{2} \bar{p}$, où $M_{\mathrm{sgs}}^{2}=2 k_{\mathrm{sgs}} /(\gamma \bar{p} / \bar{\rho})$ est le nombre de Mach de sous-maille. Par conséquent, pour des écoulements subsoniques, on supposera dans nos calculs que la partie isotrope du tenseur de sous-maille est dominée par la pression.

Il existe beaucoup d' autres modèles de sous-maille généralement plus complexes [14]. Il faut cependant souligner le fait que d'une part, ce problème reste très ouvert pour les écoulements compressibles à des nombres de Reynolds intéressants, i.e. de l'ordre de $10^{4}$ à $10^{5}$, et que d'autre part, l'objectif de ce travail n'est pas d'évaluer les modèles de sous-maille, mais de pouvoir effectuer des simulations sur des temps suffisamment longs en ayant un champ compressible exploitable acoustiquement. Dans la suite, les grandeurs ne seront plus notées ${ }^{\sim}$, bien qu'il s'agisse de quantités filtrées.

Les flux Eulériens sont très importants en aéroacoustique puisqu'ils assurent à la fois la génération et la propagation des ondes acoustiques. Ils sont donc discrétisés à l'aide d'un schéma numérique très précis, ici le schéma DRP de Tam et Webb [15] construit pour minimiser les taux de dissipation et de dispersion numériques. Ce schéma numérique a besoin de seulement 6 points par longueur d'onde pour propager efficacement les ondes acoustiques. L'intégration temporelle est effectuée par un algorithme de Runge-Kutta d'ordre 4, qui nécessite un espace mémoire réduit, et est stable jusqu'à un nombre de CFL défini par

$$
\mathrm{CFL}=\min \left\{\frac{c}{1+M} \frac{\Delta t}{\Delta x}, \frac{c \Delta t}{\Delta y}\right\}
$$

de 1,73 , où $c$ est la vitesse du son, $M$ le nombre de Mach dans la direction de l'écoulement, $\Delta t, \Delta x$ and $\Delta y$ les pas de discrétisation temporel et spatiaux. Les flux visqueux sont discrétisés à 1'aide d'un schéma aux différences finies d'ordre 2 et sont intégrés à la dernière étape de l'algorithme de Runge-Kutta. La viscosité numérique sélective de Tam [3] est également implémentée, pour dissiper les oscillations numériques haute fréquence non supportées par le schéma numérique et générées par les conditions aux limites ou l'étirement du maillage.

Finalement, le système d'équations (1) est avancé en temps de la manière suivante :

$$
\begin{aligned}
\mathbf{U}_{i, j}^{1} & =\mathbf{U}_{i, j}^{n}+\alpha_{1} \Delta t \mathbf{K e}_{i, j}^{n} \\
\mathbf{U}_{i, j}^{2} & =\mathbf{U}_{i, j}^{n}+\alpha_{2} \Delta t \mathbf{K} \mathbf{e}_{i, j}^{1} \\
\mathbf{U}_{i, j}^{3} & =\mathbf{U}_{i, j}^{n}+\alpha_{3} \Delta t \mathbf{K e}_{i, j}^{2} \\
\mathbf{U}_{i, j}^{n+1} & =\mathbf{U}_{i, j}^{n}+\alpha_{4} \Delta t \mathbf{K} \mathbf{e}_{i, j}^{3}+\Delta t \mathbf{K} \mathbf{v}_{i, j}^{n}+\Delta t \mathbf{K} \mathbf{d}_{i, j}^{n}
\end{aligned}
$$

où $\mathbf{K e}$ et $\mathbf{K v}$ sont les termes d'intégration des flux Eulériens et visqueux dont les contributions axiales, à titre d'illustrations, s'écrivent :

$$
\begin{aligned}
\mathbf{K e} \mathbf{e}_{i, j}^{k} & =-\frac{1}{\Delta x} \sum_{l=-3}^{3} a_{l} \mathbf{F e}_{i+l, j}^{k} \\
\mathbf{K} \mathbf{v}_{i, j}^{n} & =\frac{1}{2 \Delta x}\left(\mathbf{F} \mathbf{v}_{i+1, j}-\mathbf{F} \mathbf{v}_{i-1, j}\right)
\end{aligned}
$$

Kd est le terme de viscosité numérique ajouté au système (1) pour filtrer les fluctuations. Il s'écrit dans la direction axiale :

$$
\mathbf{K} \mathbf{d}_{i, j}^{n}=-\frac{c}{R_{\mathrm{s}} \Delta x} \sum_{l=-3}^{3} d_{l}\left(\mathbf{U}_{i+l, j}^{n}-\overline{\mathbf{U}}_{i+l, j}\right)
$$

où $R_{\mathrm{S}}$ est le nombre de Reynolds du schéma, habituellement de l'ordre de $R_{\mathrm{S}}=5$ et $\overline{\mathbf{U}}$ la moyenne temporelle du vecteur inconnu $\mathbf{U}$.

\section{TRAITEMENT DES CONDITIONS AUX LIMITES}

Le traitement des conditions aux limites est primordial en aéroacoustique numérique. En effet, il doit per- 
mettre la sortie des fluctuations du domaine de calcul en minimisant les éventuelles ondes acoustiques réfléchies qui viendraient contaminer le champ acoustique physique. De nombreuses formulations ont été testées afin de retenir la plus performante. Les conditions anéchoïques de Tam et Dong [16] ont finalement été implantées. Leur formulation bidimensionnelle permet d'obtenir une bien meilleure précision que les différentes méthodes basées sur les équations caractéristiques, en particulier pour des incidences obliques. Elles sont appliquées sur trois points, avec des schémas DRP décentrés, et sont integrées en temps avec le même algorithme de RungeKutta que les points intérieurs.

Des conditions aux limites de rayonnement sont utilisées aux frontières pour lesquelles les fluctuations qui sortent du domaine de calcul sont uniquement de nature acoustique. Elles sont déduites des équations d'Euler linéarisées autour du champ de vitesse moyen, dont on utilise la solution asymptotique obtenue en champ lointain pour des perturbations acoustiques. Elles s'expriment en coordonnées polaires $(r, \theta)$ par :

$$
\frac{1}{V_{\mathrm{g}}} \frac{\partial}{\partial t}\left(\begin{array}{c}
\rho \\
u_{1} \\
u_{2} \\
p
\end{array}\right)+\left(\frac{\partial}{\partial r}+\frac{1}{2 r}\right)\left(\begin{array}{c}
\rho-\bar{\rho} \\
u_{1}-\bar{u}_{1} \\
u_{2}-\bar{u}_{2} \\
p-\bar{p}
\end{array}\right)=0
$$

où $\bar{\rho}, \bar{u}_{1}, \bar{u}_{2}, \bar{p}$ sont respectivement la masse volumique moyenne, les deux composantes de la vitesse moyenne, et la pression moyenne. $V_{\mathrm{g}}$ est la vitesse de groupe des ondes acoustiques [16].

Des conditions aux limites avec sortie de fluide sont également nécessaires. Dans ce cas, on suppose que les perturbations qui atteignent la frontière du domaine sont de nature acoustique, tourbillonnaire et entropique. L'équation qui régit les fluctuations de pression, de nature acoustique si la linéarisation autour du champ moyen est valide, est inchangée, mais les trois autres sont modifiées pour permettre la sortie des fluctuations aérodynamiques convectées par l'écoulement moyen. On utilise donc le système suivant :

$$
\left\{\begin{array}{l}
\frac{\partial \rho}{\partial t}+\overline{\mathbf{u}} \cdot \nabla(\rho-\bar{\rho})=\frac{1}{\bar{c}^{2}}\left(\frac{\partial p}{\partial t}+\overline{\mathbf{u}} \cdot \nabla(p-\bar{p})\right) \\
\frac{\partial u_{1}}{\partial t}+\overline{\mathbf{u}} \cdot \nabla\left(u_{1}-\bar{u}_{1}\right)=-\frac{1}{\bar{\rho}} \frac{\partial(p-\bar{p})}{\partial x} \\
\frac{\partial u_{2}}{\partial t}+\overline{\mathbf{u}} \cdot \nabla\left(u_{2}-\bar{u}_{2}\right)=-\frac{1}{\bar{\rho}} \frac{\partial(p-\bar{p})}{\partial y} \\
\frac{1}{V_{\mathrm{g}}} \frac{\partial p}{\partial t}+\frac{\partial(p-\bar{p})}{\partial r}+\frac{(p-\bar{p})}{2 r}=0
\end{array}\right.
$$

où $\bar{c}=\sqrt{\gamma \bar{p} / \bar{\rho}}$ est la vitesse moyenne du son.
La précision de ces conditions aux limites a été évaluée avec les cas tests du premier Workshop de l'ICASE [17], i.e. les cas d'une impulsion acoustique et d'un tourbillon en présence d'un écoulement moyen. La sortie du domaine de calcul d'un tourbillon produit des ondes acoustiques parasites, qui sont faibles par rapport aux fluctuations aérodynamiques incidentes, mais qui risquent de ne pas être négligeables vis à vis des ondes acoustiques physiques en raison de la différence de grandeur entre les phénomènes aérodynamiques et acoustiques. On utilise donc en sortie d'écoulement une zone éponge construite à l'aide de deux méthodes. Tout d'abord le maillage est étiré afin de dissiper les structures tourbillonnaires avant qu'elles n'atteignent la frontière aval. Un terme de dissipation de type PML (pour perfectly matched layer [18]) est également ajouté aux équations pour éviter la propagation d'ondes réfléchies éventuellement générées par la zone éponge. Enfin, les flux visqueux sont résolus aux frontières amont et aval, afin d'éviter toute discontinuité de ces termes.

\section{SIMULATION D'UNE COUCHE DE MÉLANGE}

On considère une couche de mélange bidimensionnelle présentant en entrée un profil de vitesse longitudinale en tangente hyperbolique de la forme:

$$
u_{1}(y)=\frac{U_{1}+U_{2}}{2}+\frac{U_{2}-U_{1}}{2} \tanh \left(\frac{2 y}{\delta_{\omega}(0)}\right)
$$

où $U_{1}$ et $U_{2}$ sont respectivement les vitesses des écoulements lent et rapide, et $\delta_{\omega}(0)$ l'épaisseur de vorticité initiale de la couche de mélange. Les vitesses ont été fixées à $U_{1}=0,12 c_{0}$ et $U_{2}=0,48 c_{0}$, donnant un nombre de Reynolds basé sur l'épaisseur de vorticité de $R e_{\omega}=$ $\delta_{\omega}(0)\left(U_{2}-U_{1}\right) / v=12800$.

Afin d'amorcer et de contrôler le développement des structures turbulentes, des perturbations de vitesse sont ajoutées en entrée d'écoulement. Elles sont de type incompressible pour ne pas générer d'ondes acoustiques parasites. La couche de mélange est excitée à la fréquence $f_{0}$ et au premier sous-harmonique $f_{0} / 2$. La fréquence fondamentale $f_{0}$ correspond au plus fort taux d'amplification des instabilités associées à un profil de vitesse en tangente hyperbolique, et est calculée par la théorie de l'instabilité linéaire [19]. On fixe ainsi le lieu des appariements vers $x=70 \delta_{\omega}(0)$, comme on le constate sur la figure 2. La fréquence des appariements est alors de $f_{\mathrm{p}}=f_{0} / 2$. 


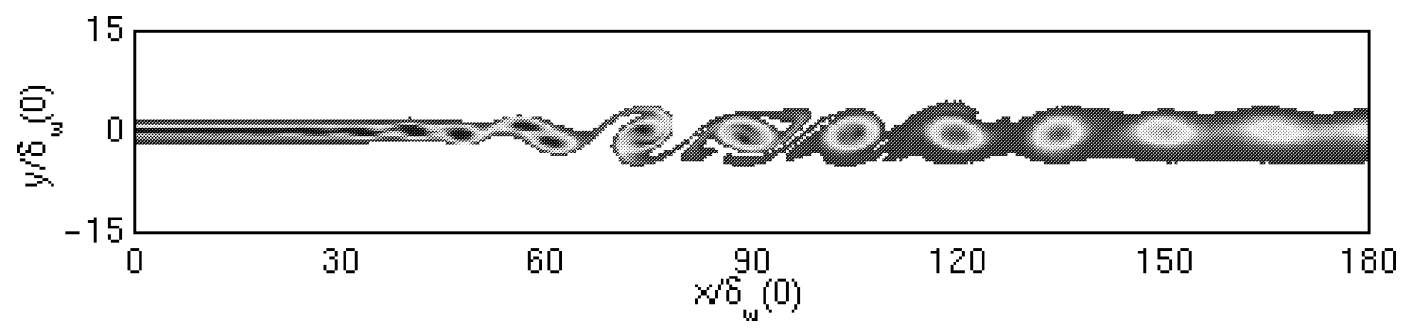

Figure 2. Représentation de la vorticité obtenue en excitant la couche de mélange à $f_{0}$ et $f_{0} / 2$. Les niveaux de vorticité vont de $-45 \cdot 10^{4}$ à $-2 \cdot 10^{4} \mathrm{~s}^{-1}$.

Figure 2. Snapshot of the vorticity field computed by exciting the mixing layer at $f_{0}$ and $f_{0} / 2$. The vorticity levels are defined from $-45 \cdot 10^{4}$ to $-2 \cdot 10^{4} \mathrm{~s}^{-1}$.

Le maillage est constitué de $440 \times 440$ mailles. Une zone éponge est construite en sortie d'écoulement au niveau de la zone de cisaillement. Elle dissipe les tourbillons à partir de $x=130 \delta_{\omega}(0)$ et interdit d'autres appariements en aval.

Colonius et al. [9] ont montré numériquement que les appariements sont à l'origine, dans une couche de mélange entre deux fluides à Mach 0,25 et 0,5 , d'un rayonnement à la fréquence $f_{\mathrm{p}}$. Pour cela ils ont dû filtrer le résultat de leur simulation numérique directe précisément à cette fréquence. Dans notre étude, la fréquence d'excitation est égale à $f_{0}=8250 \mathrm{~Hz}$, et une longueur d'onde acoustique $\lambda_{f_{\mathrm{p}}}=51,5 \delta_{\omega}(0)$ est donc attendue. On représente sur la figure 3 la dilatation $\Theta=$ $\nabla \cdot \mathbf{u}$, calculée sur tout le maillage. Des fronts d'ondes provenant de la zone des appariements apparaissent très nettement [20], et présentent une longueur d'onde en accord avec la fréquence $f_{\mathrm{p}}$. La propagation acoustique subit les effets de convection par les écoulements, lent en bas et rapide en haut. Ils se traduisent par une ovalisation des fronts d'ondes, qui est surtout marquée sur la partie haute. Les niveaux de dilatation sont de l'ordre de $2 \mathrm{~s}^{-1}$, correspondant à des fluctuations de pression acoustique de $10 \mathrm{~Pa}$ environ. Cette valeur est petite par rapport aux fluctuations de pression de type aérodynamique qui sont supérieures à $1000 \mathrm{~Pa}$. Le rayonnement acoustique est plus prononcé dans la direction aval, et en particulier pour des angles proches de $\theta_{1} \approx-70^{\circ}$ pour le fluide du bas et $\theta_{2} \approx 60^{\circ}$ pour le fluide du haut. La différence entre ces deux directivités peut également être attribuée aux effets de convection.

Enfin, la figure 4 représente le profil de dilatation en $x=70 \delta_{\omega}(0)$ dans la direction perpendiculaire à l'écoulement, i.e. à hauteur de la zone des appariements. On observe la décroissance des ondes acoustiques depuis la source de bruit, avec une longueur d'onde égale à $\lambda_{f_{\mathrm{p}}}$. L'enveloppe du profil de dilatation est approchée par

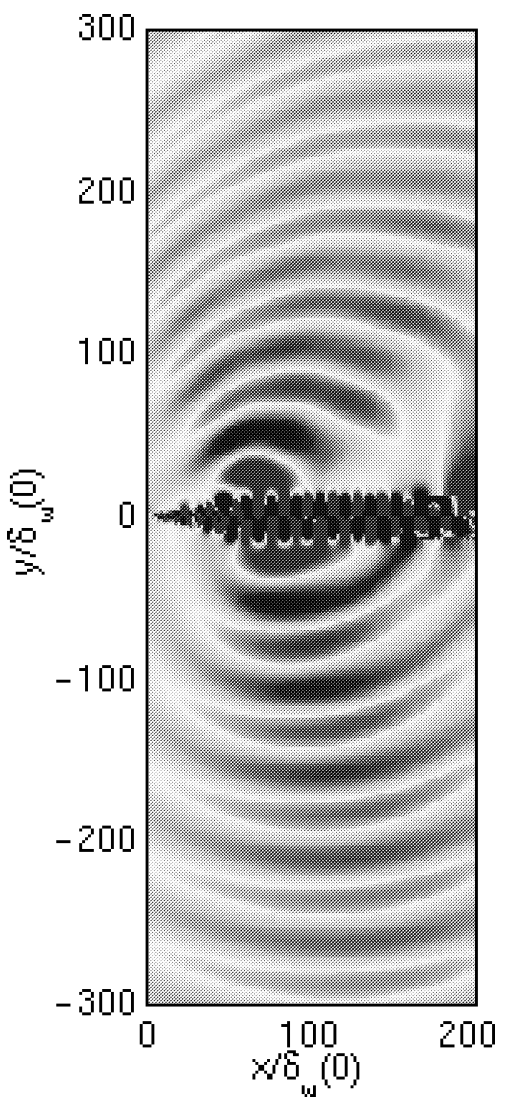

Figure 3. Représentation du champ de dilatation $\Theta=\nabla \cdot \mathbf{u}$ obtenu sur tout le domaine de calcul; les niveaux vont de $-1,6$ à $1,6 \mathrm{~s}^{-1}$.

Figure 3. Snapshot of the dilatation field $\Theta=\nabla \cdot \mathbf{u}$ computed on the whole computational domain. The dilatation levels are defined from -1.6 to $1.6 \mathrm{~s}^{-1}$.

une courbe proportionnelle à $1 / \sqrt{y}$. On retrouve ici la décroissance acoustique classique en $1 / \sqrt{r}$ obtenue en champ lointain bidimensionnel. 


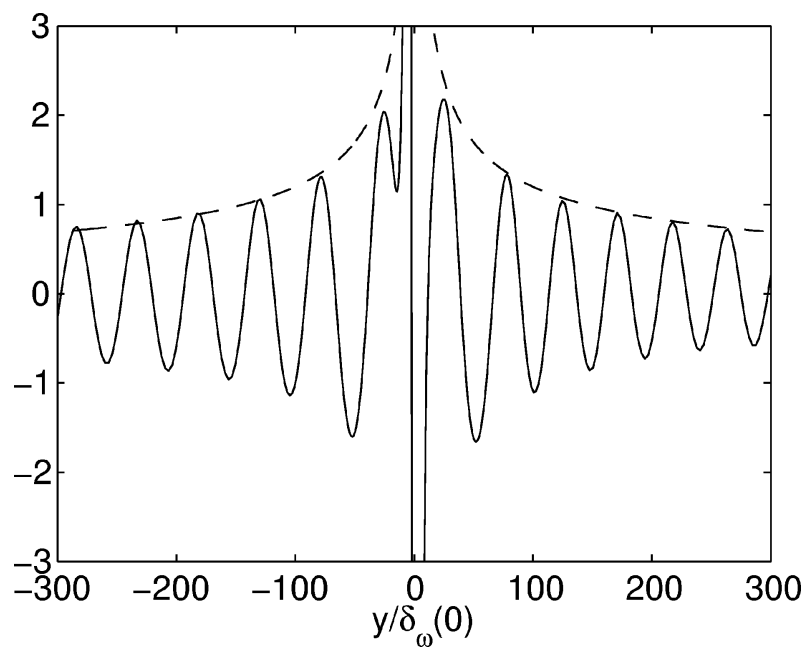

Figure 4. Profil de dilatation défini en $x=70 \delta_{\omega}(0)$. Les tirets représentent une courbe décroissant en $1 / \sqrt{y}$. La dilatation est exprimée en $\mathrm{s}^{-1}$.

Figure 4. Dilatation profile defined at $x=70 \delta_{\omega}(0)$. Dashed line represents a $1 / \sqrt{y}$ decay. The dilatation levels are given in $\mathrm{s}^{-1}$.

\section{MÉCANISME DE GÉNÉRATION DE BRUIT PAR APPARIEMENTS}

La figure 5 présente la zone des appariements à quatre instants successifs. Les images sont régulièrement réparties sur la période $T_{\mathrm{p}}=1 / f_{\mathrm{p}}$, correspondant à l'intervalle de temps entre deux fusions successives. Le cycle (a)-(b)-(c)-(d)-(a) décrit donc une période complète. Le champ de vorticité est superposé sur le champ de dilatation au niveau de la zone de cisaillement, afin de mettre en évidence le mécanisme de bruit associé à l'appariement de tourbillons dans une couche de mélange.

On observe une structure en double hélice du champ de dilatation, centrée sur l'appariement de deux tourbillons, qui est particulièrement visible sur la figure 5(b). Cette caractéristique a précédemment été mise en évidence dans le cas de deux tourbillons co-rotatifs sur la figure 1. On identifie ce mécanisme de génération de bruit comme un quadrupôle tournant produit par l'enroulement des deux tourbillons [6].

On peut décrire plus en détail les différentes étapes d'un appariement. Sur la figure 5(a), deux tourbillons successifs se sont rapprochés pour se retrouver côte à côte. Ils commencent à s'enrouler l'un autour de l'autre, ce qui marque le début du rayonnement acoustique, avec un premier lobe de rayonnement très sombre. Sur la $f i$ gure 5(b), un second lobe, plus clair, correspondant au second bras du quadupôle, apparaît perpendiculairement au premier. Le premier lobe arrête de rayonner et c'est le se- cond qui assure une émision acoustique sur la figure 5(c). Enfin, le second lobe plus clair arrête d'émettre à son tour sur la figure 5(d). Sur la figure 5(a), les deux tourbillons ont complètement fusionné, tandis qu'un nouvel appariement débute.

L'étude de Mitchell et al. [21] consacrée aux tourbillons co-rotatifs a montré que l'appariement des deux tourbillons produit un rayonnement acoustique pendant la période de rotation. L'amplitude du rayonnement acoustique atteint un pic au moment de la coalescence des deux tourbillons pour diminuer de manière significative après leur fusion.

Dans le cas de la couche de mélange, la durée d'émission acoustique associée à une paire de tourbillons est égale à une période d'appariement $T_{\mathrm{p}}$. Ainsi, les fronts d'ondes produits par les appariements successifs s'assemblent parfaitement. On observe en particulier qu'il n'y a pas de discontinuité dans le champ acoustique, car une discontinuité impliquerait une durée d'émission plus petite que la période d'appariement, i.e. l'existence d'une période de transition pendant laquelle il n'y a plus de génération de bruit. Inversement, les rayonnements de deux appariements ne peuvent pas interférer, puisque des interférences apparaitraient seulement si deux sources de bruit rayonnaient simultanément, soit pour une durée d'émission plus grande que la période d'appariement.

L'absence d'interférence est également liée au faible déplacement des sources acoustiques. Celui-ci n'intervient que sur une très courte distance, environ $5 \delta_{\omega}(0)$, pendant l'appariement. Les deux fronts d'ondes produits par un appariement semblent d'ailleurs provenir de deux lieux très proches. Sur la figure 3 , les fronts d'ondes ne sont pas exactement concentriques, les fronts les plus sombres ayant été émis légérement plus en amont.

De plus, la longueur d'onde acoustique est directement associée à la vitesse de rotation des deux tourbillons. Pour produire un rayonnement acoustique avec une longueur d'onde donnée par la fréquence d'appariement $f_{\mathrm{p}}$, la vitesse de rotation doit correspondre à la moitié de cette fréquence, en raison de la symétrie de la source quadrupolaire. En d'autres termes, les tourbillons accomplissent un demi-tour pendant la période d'appariement $T_{\mathrm{p}}$. Cette propriété est illustrée sur les représentations du champ de vorticité de la figure 6. L'utilisation d'iso-contours permet de détailler l'orientation des deux tourbillons qui s'apparient, aux quatre instants définis sur la figure 5. Les tourbillons accomplissent un huitième de tour tous les $T_{\mathrm{p}} / 4$. La figure $6(d)$ présente également, côte à côte, les deux orientations des tourbillons avant et après la période $T_{\mathrm{p}}$ d'appariement, et ceux-ci ont bien réalisé un demi-tour. 
(a)

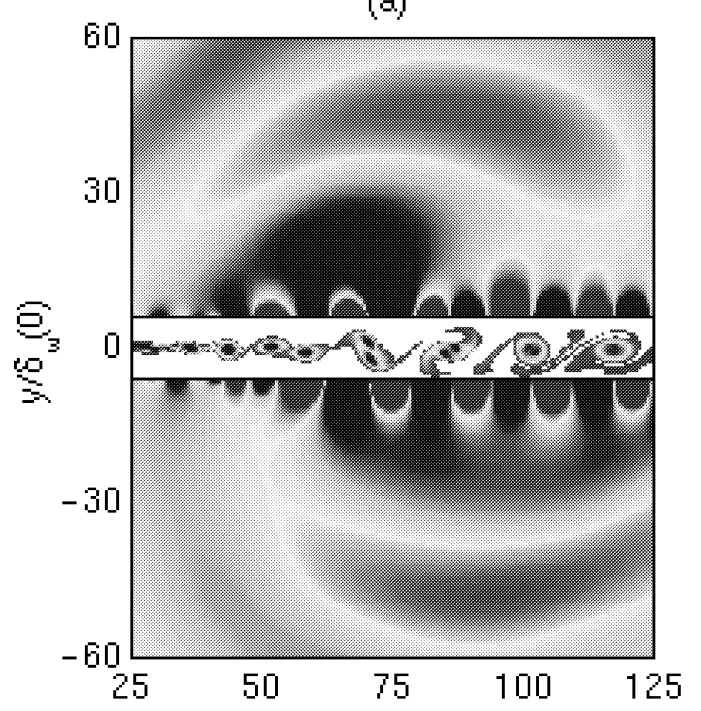

(c)

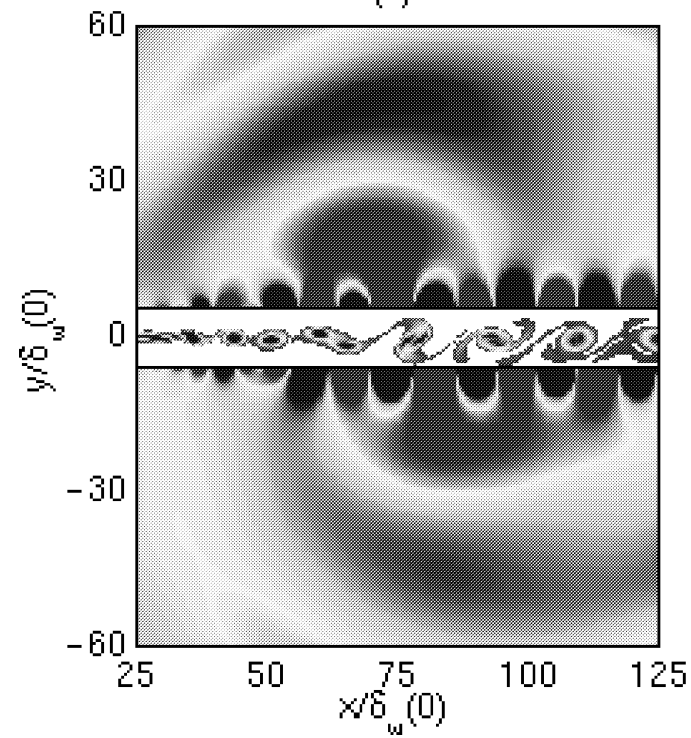

(b)

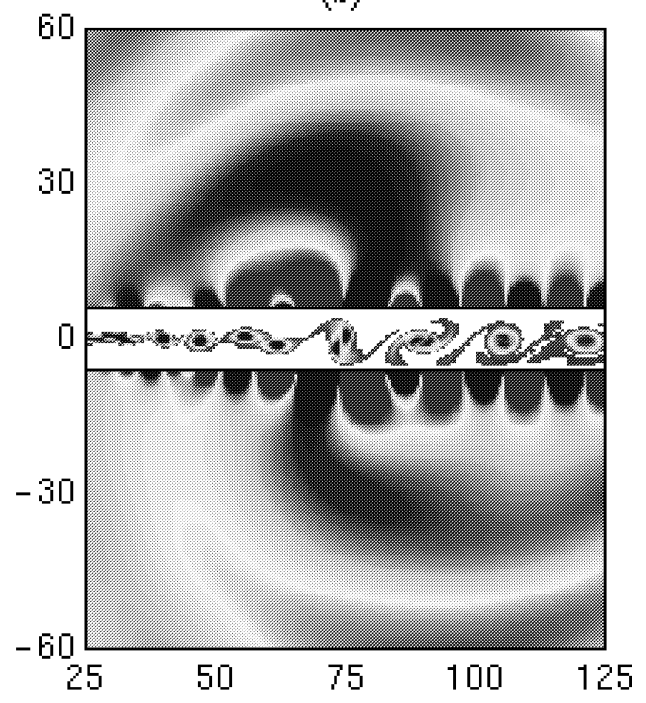

(d)

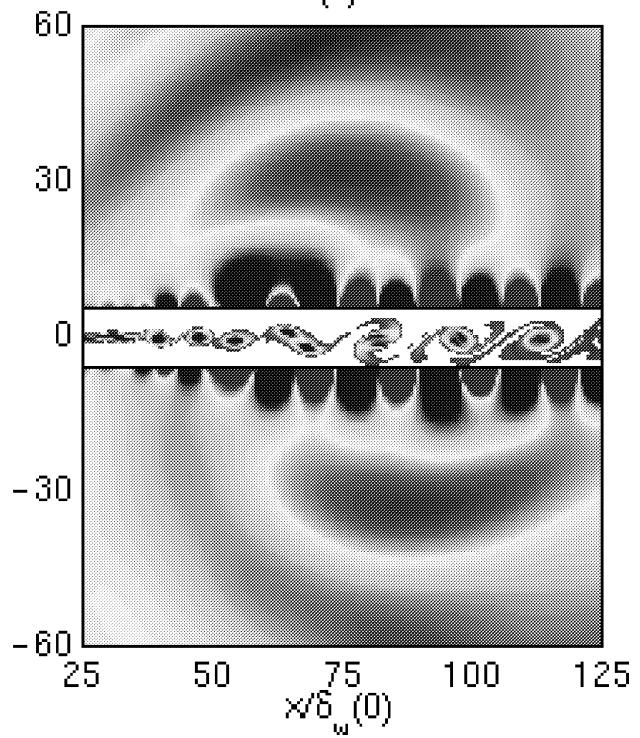

Figure 5. Quatre représentations de la zone des appariements avec le champ de vorticité au centre de l'écoulement et le champ de dilatation ailleurs. L'intervalle de temps entre deux figures successives est de $T_{\mathrm{p}} / 4$. Le cycle (a)-(b)-(c)-(d)-(a) décrit donc une période d'appariement. Les niveaux de dilatation vont de -3 à $3 \mathrm{~s}^{-1}$. Les niveaux de vorticité sont identiques à ceux de la figure 2 .

Figure 5. Four snapshots of the pairing zone with the vorticity field in the flow region and the dilatation field all around. The time between two successives pictures is $T_{\mathrm{p}} / 4$. A pairing period is also described by the cycle (a)-(b)-(c)-(d)-(a). The dilatation levels are defined from -3 to $3 \mathrm{~s}^{-1}$. The vorticity levels are those of the figure 2.

La régularité du champ acoustique est donc due à la dynamique de l'appariement. Les appariements se succèdent au même endroit tous les $T_{\mathrm{p}}$, et chacun des appariements génère un rayonnement à la fréquence $f_{\mathrm{p}}$ pendant la période $T_{\mathrm{p}}$. Les effets de réfraction pourraient également être invoqués pour expliquer la structure du rayonnement acoustique. Cependant, en raison de la grande différence entre l'épaisseur de cisaillement et la longueur d'onde acoustique, on peut s'attendre à ce que la réfraction soit peu importante $[7,10]$. 
(a)

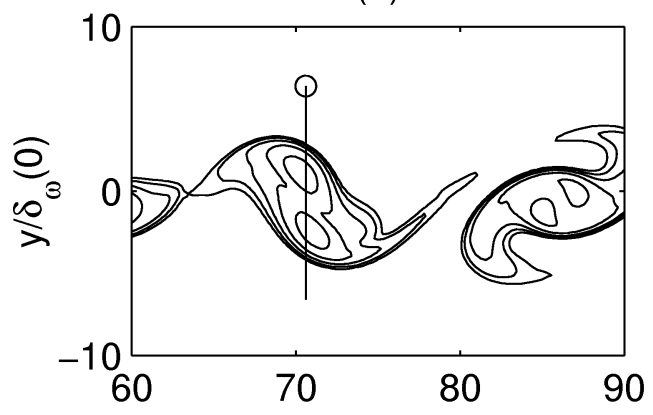

(c)

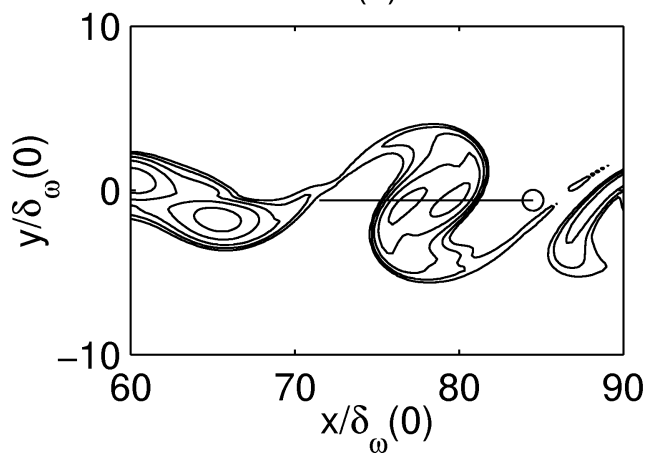

(b)

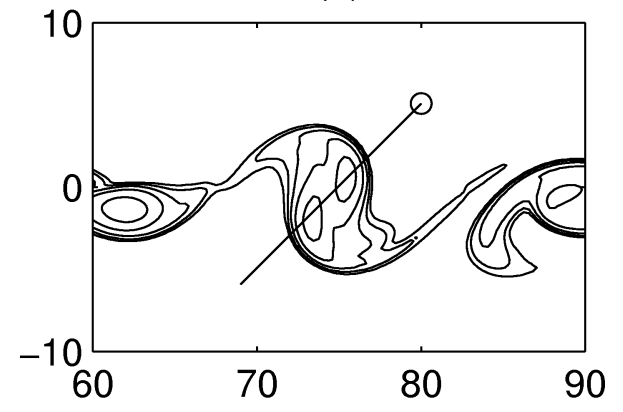

(d)

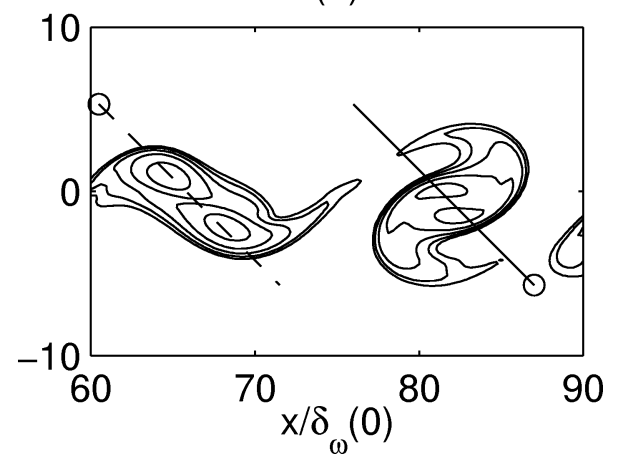

Figure 6. Quatre représentations de la vorticité au niveau de la zone des appariements aux même instants que sur la figure 5. Cinq iso-contours allant de -250 à $-4 \cdot 10^{4} \mathrm{~s}^{-1}$ suivant une progression géométrique de raison 2 . $\longrightarrow$, orientation des deux tourbillons co-rotatifs ; - - - , orientation des tourbillons suivants.

Figure 6. Four snapshots of the vorticity field in the pairing region at the four times of figure 5 . The five isolines of vorticity are defined from -250 to $-4 \cdot 10^{4} \mathrm{~s}^{-1}$ following a geometrical ratio of 2 . $\longrightarrow$, orientation of two co-rotative vortices; - - - , orientation of the next vortices.

\section{CONCLUSION ET PERSPECTIVES}

Le calcul direct du rayonnement acoustique d'une couche de mélange bidimensionnelle a été réalisé avec succès. Il nous a permis de mettre en évidence le rôle des appariements de tourbillons dans la génération de bruit. Les appariements successifs produisent un rayonnement acoustique régulier à la fréquence des appariements. Le champ acoustique obtenu directement par SGE peut également servir de solution de référence afin d'évaluer la précision des méthodes hybrides, comme l'analogie de Lighthill [10] ou les équations d'Euler linéarisées [22] .

Le calcul direct du rayonnement acoustique à partir des équations de Navier-Stokes est une démarche récente, mais qui est amenée à se développer rapidement. L'utilisation de la Simulation des Grandes Échelles permettra notamment de l'appliquer à des écoulements pré- sentant des nombres de Reynolds réalistes, qui se rapprochent de configurations industrielles. Le cas d'un jet turbulent 3-D pleinement développé avec un nombre de Mach de 0,9 et un nombre de Reynolds de $6,5 \cdot 10^{4}$ a été étudié tout récemment [11]. La comparaison avec les expériences est alors possible, et les premiers résultats sont en bon accord en terme de niveaux de pression, de spectres et de directivité.

\section{Remerciements}

Ce travail a été réalisé avec le soutien financier d'Électricité de France. Les calculs ont été effectués sur le Cray C-98 du Centre National de la Recherche Scientifique, mis à disposition par l'IDRIS (Institut de Développement et des Ressources en Informatique Scientifique). 


\section{RÉFÉRENCES}

[1] Lighthill M.J., On sound generated aerodynamically, I. General theory, Proc. Roy. Soc. London Ser. A 211 A1107 (1952) 564-587.

[2] Bailly C., Lafon P., Candel S., Subsonic and supersonic jet noise prediction from statistical source models, AIAA J. 35 (11) (1997) 1688-1696.

[3] Tam C.K.W., Computational aeroacoustics : issues and methods, AIAA J. 33 (10) (1995) 1788-1796.

[4] Freund J.B., Lele S.K., Moin P., Direct simulation of a Mach 1.92 jet and its sound field, in : Proceedings of 4th AIAA/CEAS Aeroacoustics Conference, 1998, AIAA Paper 98-2291.

[5] Freund J.B., Acoustic sources in a turbulent jet : a direct numerical simulation study, in : Proceedings of 5th AIAA/CEAS Aeroacoustics Conference, 1999, AIAA Paper 99-1858.

[6] Powell A., Theory of vortex sound, J. Acoust. Soc. Amer. 36 (1) (1964) 177-195.

[7] Bogey C., Calcul direct du bruit aérodynamique et validation de modèles acoustiques hybrides, Thèse, $\mathrm{n}^{\mathrm{o}} 2000-11$, École Centrale de Lyon, 2000.

[8] Winant C.D., Browand F.K., Vortex pairing : the mechanism of turbulent mixing layer growth at moderate Reynolds number, J. Fluid Mech. 63 (2) (1974) 237-255.

[9] Colonius T., Lele S.K., Moin P., Sound generation in a mixing layer, J. Fluid Mech. 330 (1997) 375-409.

[10] Bogey C., Bailly C., Juvé D., Computation of mixing layer noise using large eddy simulation, in : Proceedings of 5th AIAA/CEAS Aeroacoustics Conference, 1999, AIAA Paper 99-1871 (à paraître dans l'AIAA J.).

[11] Bogey C., Bailly C., Juvé D., Computation of the sound radiated by a 3-D jet using large eddy simulation, in :
Proceedings of 6th AIAA/CEAS AeroAcoustics Conference, 2000, AIAA Paper 2000-2009.

[12] Smagorinsky J.S., General circulation experiments with the primitive equations. I. The basic experiment, Mon. Weath. Rev. 91 (1963) 99-163.

[13] Erlebacher G., Hussaini M.Y., Speziale C.G., Zang T.A., Toward the large-eddy simulation of compressible turbulent flows, J. Fluid Mech. 238 (1992) 155-185.

[14] Lesieur M., Métais O., New trends in large-eddy simulations of turbulence, Annual Rev. Fluid Mech. 28 (1996) 45-82.

[15] Tam C.K.W., Webb J.C., Dispersion-relation-preserving finite difference schemes for computational acoustics, J. Comput. Phys. 107 (1993) 262-281.

[16] Tam C.K.W., Dong Z., Radiation and outflow boundary conditions for direct computation of acoustic and flow disturbances in a nonuniform mean flow, J. Comput. Acoust. 4 (2) (1996) 175-201.

[17] Hardin J.C., Ristorcelli J.R., Tam C.K.W. (Eds.), ICASE/ NASA, Workshop on Benchmark Problems in Computational Aeroacoustics, NASA CP 3300, 1995.

[18] Bérenger J.-P., A perfectly matched layer for the absorption of electromagnetic waves, J. Comput. Phys. 114 (1994) 185-200.

[19] Michalke A., On the inviscid instability of the hyperbolictangent velocity profile, J. Fluid Mech. 19 (1964) 543-556.

[20] Bogey C., Bailly C., Juvé D., Calcul direct du rayonnement acoustique d'une couche de mélange par macrosimulation, C. R. Acad. Sci. Paris Sér. IIb 327 (1999) 1029-1034.

[21] Mitchell B.E., Lele S.K., Moin P., Direct computation of the sound from a compressible co-rotating vortex pair, J. Fluid Mech. 285 (1995) 181-202.

[22] Bogey C., Bailly C., Juvé D., Calcul du rayonnement acoustique d'une couche de mélange à l'aide des équations d'Euler linéarisées, C. R. Acad. Sci. Paris Sér. IIb 328 (2000) 341-347.

\section{Abridged English version \\ Noise generation by vortex pairings in shear flows}

In order to reduce the noise radiated by turbulent flows, numerical methods capable of predicting aerodynamic sound with accuracy are required. Initially, hybrid methods such as the Lighthill analogy [1] were used. They consist of two-step calculations separating noise generation and propagation. Indeed, the aerodynamic field is determined by a first computation, then acoustic source terms are built up and applied in suitable sound propagation techniques to calculate the noise radiation. Restrictive hypotheses are used in the modelling of source terms, and constitute the main failure of this approach. Moreover, acoustic-flow interactions are often not taken into account correctly in the wave operator. A recent alternative of hybrid methods is the direct computation of acoustic radiation. Both aerodynamic perturbations and acoustic waves are determined directly by solving the Navier-Stokes equations. However, this direct approach has to face serious numerical issues owing to the great disparity of scales and levels between the acoustic and aerodynamic fields, which have led to the development of techniques specific to computational aeroacoustic [3].

The goal of this study is to calculate directly the acoustic field generated by vortex pairings in a mixing layer, in order to provide a better understanding of this typical subsonic noise mechanism in shear flows. A LES code (ALESIA for Appropriate Large Eddy SImulation for Aeroacoustics) developed especially for aeroacoustic simulations is used [7, 10]. Only larger turbulent scales are calculated, while the effects of smaller ones are taken into account by means of the subgrid scale 
model of Smagorinsky [12]. The DRP scheme of Tam and Webb [15] is used for spatial discretization, and time advancement is carried out by a fourth-order step RungeKutta algorithm. This algorithm is highly stable, and requires low memory storage. The boundary conditions of Tam and Dong [16] are implemented. They are based on the asymptotic solution of linearized Euler's equations in the acoustic far-field. The generation of spurious acoustic waves is also minimized thanks to a sponge zone added at the outflow boundary. This zone is built with the combination of the perfectly matched layer idea of Berenger [18] and a grid stretching.

Vortex pairing is a well-known source of sound in subsonic low Reynolds number flows. The source behaves like a rotating quadrupole according to the works of Powell [6] and Mitchell [21]. Here a LES of sound generation by vortex pairing is reported.

A two-dimensional mixing layer between two parallel flows with respective speeds of $0.12 c_{0}$ and $0.48 c_{0}$ is simulated by LES. The Reynolds number based on the initial vorticity thickness $\delta_{\omega}(0)$ is equal to 12800 . The fundamental frequency of the mixing layer $f_{0}$ is calculated by the linear instability theory [19]. It corresponds to the highest amplification rate of instabilities associated with the tangent-hyperbolic velocity profile. The mixing layer is excited at its fundamental frequency $f_{0}$ and its first subharmonic $f_{0} / 2$, in order to control the pairings of vortices. Indeed, their locations are fixed around $x=70 \delta_{\omega}(0)$, as shown by the vorticity field in figure 2. The dilatation field computed on the whole computational domain is displayed in figure 3. Pairings generate an acoustic radiation at the frequency of pairing $f_{\mathrm{p}}=f_{0} / 2$, as found in DNS by Colonius [9]. The wave fronts are visibly modified owing to the convection effects induced by the rapid upper flow. The generation mechanism is also displayed in figure 5 made up of four snapshots of the pairing region regularly spread over a pairing period $T_{\mathrm{p}}=1 / f_{\mathrm{p}}$. The vorticity field is superimposed on the dilatation field on the shear layer. A double spiral pattern is observed, particularly in figure 5(b). Previous works on co-rotative vortices $[6,21]$ have identified this source as a rotating quadrupole with four lobes. Furthermore, emission time associated to a vortex pair lasted for a pairing period $T_{\mathrm{p}}$. Thus, the subsequent pairing radiations are perfectly matched. Finally, acoustic wavelength is directly connected to the frequency of rotation of the two vortices. This is illustrated by figure 6 representing the vorticity field, and more precisely the successive orientations of the vortex pair at the four times defined in figure 5. The vortex pair completes half a revolution during a period $T_{\mathrm{p}}$, as shown in figure $6(d)$ displaying side by side the two opposite orientations of the vortex pair before and after a period $T_{\mathrm{p}}$. So, the rotating frequency corresponds to $f_{\mathrm{p}} / 2$, which leads to the acoustic frequency $f_{\mathrm{p}}$ owing to the symmetry of the quadrupolar source.

This preliminary study shows the feasibility of direct calculation of the acoustic radiation using large eddy simulation. This direct approach can now be applied to three-dimensional flows, such as round jets $[7,11]$. 